\title{
One-Shot Manipulation of Dynamical Quantum Resources
}

\author{
Bartosz Regula $\oplus^{1, *, \dagger}$ and Ryuji Takagi $\oplus^{1,2, *, *}$ \\ ${ }^{1}$ School of Physical and Mathematical Sciences, Nanyang Technological University, 637371, Singapore \\ ${ }^{2}$ Center for Theoretical Physics and Department of Physics, Massachusetts Institute of Technology, \\ Cambridge, Massachusetts 02139, USA
}

(Received 29 January 2021; accepted 21 June 2021; published 5 August 2021)

\begin{abstract}
We develop a unified framework to characterize one-shot transformations of dynamical quantum resources in terms of resource quantifiers, establishing universal conditions for exact and approximate transformations in general resource theories. Our framework encompasses all dynamical resources represented as quantum channels, including those with a specific structure — such as boxes, assemblages, and measurements - thus immediately applying in a vast range of physical settings. For the particularly important manipulation tasks of distillation and dilution, we show that our conditions become necessary and sufficient for broad classes of important theories, enabling an exact characterization of these tasks and establishing a precise connection between operational problems and resource monotones based on entropic divergences. We exemplify our results by considering explicit applications to quantum communication, where we obtain exact expressions for one-shot quantum capacity and simulation cost assisted by nosignaling, separability-preserving, and positive partial transpose-preserving codes; as well as to nonlocality, contextuality, and measurement incompatibility, where we present operational applications of a number of relevant resource measures.
\end{abstract}

DOI: 10.1103/PhysRevLett.127.060402

Introduction.-Manipulating different resources under physical restrictions underpins many quantum technologies, and the precise understanding of physically realizable transformations is a fundamental question both from theoretical and practical points of view. Quantum resource theories [1-3] provide a platform through which the quantification and manipulation of resources can be explicitly considered, enabling the study of resource manipulation in a range of settings of interest [4-7].

Because of the inherent generality of the framework of resource theories, the formalism could be expected to provide unifying results on resource manipulation that hold for diverse classes of resources. Developing such versatile general resource theories allows one to extract common features shared by different physical phenomena and clarify the peculiarities that differentiate one setting from others [7-25]. However, previous approaches to these problems suffer from a number of limitations. Many works have focused on the static, rather than dynamical resources, in the sense that only the manipulation of quantum states was considered, and a more general approach that incorporates the ability to manipulate the dynamics of the systems was

Published by the American Physical Society under the terms of the Creative Commons Attribution 4.0 International license. Further distribution of this work must maintain attribution to the author(s) and the published article's title, journal citation, and DOI. not established. Recent works have begun to describe channel-based theories [14,22,23,26-30], but often focused on the investigation of specific theories such as entanglement, coherence, or quantum memories [31-43], or obtained results that only apply in the idealized asymptotic limit [26]. The characterization of dynamical resource theories is significantly more complex than the manipulation of underlying states, and many questions remain unanswered. Notably, the precise characterization of convertibility between two quantum channels in the practical one-shot scenario has been an outstanding problem to be addressed.

An important aspect of dynamical resources is that they can describe a much broader range of settings than the commonly considered manipulation of quantum states with general quantum channels. For instance, Bell nonlocality $[44,45]$ as well as quantum contextuality $[46,47]$ have been investigated within formal resource-theoretic settings [48-52], but the specific restrictions on the structure of channels allowed in these settings prevented them from being integrated into most of the previous general quantum resource frameworks. The recent works of Refs. [53,54] considered an approach to quantum nonlocality that encompasses any type of input and output systems, suggesting that the establishment of a broader framework of channel resource theories might be possible, and opening up the potential to address the manipulation of dynamical resources in a unified manner.

Here, we achieve such a unified description by providing a universal characterization of one-shot resource 
transformations with finite error in terms of fundamental resource measures, valid in general resource theories of quantum channels. By allowing arbitrary restrictions on the set of channels under consideration, our results apply not only to settings previously studied in resource theories of channels-e.g., entanglement, coherence, magic, quantum thermodynamics, and quantum communication-but also to other dynamical resources such as Bell nonlocality, contextuality, steering [55,56], measurement incompatibility [57], and many others [53,54], offering a very general quantitative description of the fundamental task of resource manipulation. As an important subclass of manipulation tasks, we study resource distillation and dilution, where we present additional bounds and results that provide necessary and sufficient conditions for resource conversion in many relevant cases. This provides an exact characterization of optimal one-shot rates achievable in these tasks, in which case important resource measures - such as resource robustness and hypothesis testing relative entropy-are endowed with explicit operational meaning. We further establish tight benchmarks on the achievable fidelity of distillation. Finally, we discuss insights provided by our general results into several physical scenarios. We first apply our results to quantum communication and find exact expressions for quantum capacity and simulation cost for communication assisted by no-signaling codes and codes preserving separability or the positivity of the partial transpose (PPT). We then discuss the application to nonlocality, contextuality, and measurement incompatibility, where we link resource measures previously introduced in other settings to approximate resource transformations.

We focus on discussing our main results below, and the technical proofs are deferred to the Supplemental Material [58].

Manipulation of dynamical resources.-Let $\mathbb{O}_{\text {all }}$ be the set of valid channels allowed in the given physical setting; this can be the set of all quantum channels or a subset thereof, allowing us to take into consideration possible restrictions on the types of channels being manipulated. Each resource theory also designates a subset of channels that are considered to be available for free, and we denote the given free channels as $\mathbb{O} \subseteq \mathbb{O}_{\text {all }}$. We impose mild assumptions that the underlying Hilbert spaces are always finite dimensional and, for a fixed dimension, the set of free channels $\mathbb{O}$ is convex and closed [7].

General transformations of quantum channels are described by quantum superchannels [65]. Since superchannels need not preserve specific channel structures in general [53], we consider the subset of superchannels that map the set of allowed channels $\mathbb{O}_{\text {all }}$ to allowed output channels $\mathbb{O}_{\text {all }}^{\prime}$, defined as $\mathbb{S}_{\text {all }}:=\left\{\Theta: \mathbb{O}_{\text {all }} \rightarrow \mathbb{O}_{\text {all }}^{\prime}\right\}$. We can now take a subset of $\mathbb{O}_{\text {all }}^{\prime}$ and consider it as the free channels in the output space, which we denote by $\mathbb{O}^{\prime}$. A subset of $\mathbb{S}_{\text {all }}$ serves as the set of free transformation that can be used for the manipulation of resources. The standard requirement for any free operation is that it should not generate any resource, i.e., it should not create any costly channel out of a free one. We consider the maximal set satisfying this condition, $\mathbb{S}:=\left\{\Theta \in \mathbb{S}_{\text {all }} \mid \Theta(\mathcal{M}) \in \mathbb{O}^{\prime} \quad \forall \mathcal{M} \in \mathbb{O}\right\}$.

Our goal is to find the conditions for the transformation from $\mathcal{E}$ to $\mathcal{N}$ using free superchannels in $\mathbb{S}$, given any two channels $\mathcal{E} \in \mathbb{O}_{\text {all }}$ and $\mathcal{N} \in \mathbb{O}_{\text {all }}^{\prime}$. In practice, the transformation can often only be achieved approximately, especially in nonasymptotic resource manipulation. To evaluate the inevitable error, we consider the worst-case fidelity [66,67] defined for two channels $\mathcal{E}_{1}, \mathcal{E}_{2} \in \mathbb{O}_{\text {all }}$ as $F\left(\mathcal{E}_{1}, \mathcal{E}_{2}\right):=\min _{\rho} F\left(\right.$ id $\otimes \mathcal{E}_{1}(\rho)$, id $\left.\otimes \mathcal{E}_{2}(\rho)\right)$ where $F(\rho, \sigma):=\|\sqrt{\rho} \sqrt{\sigma}\|_{1}^{2}$ is the fidelity.

Our aim will be to characterize the conditions on resource transformation through the resource contents of the given channels. To this end, we introduce two types of resource measures. They both belong to, or are closely related to, the class of one-shot entropic quantities [68] and, in particular, channel divergences $[69,70]$. The first class is known as the robustness measures [71] defined for any $\mathcal{E} \in \mathbb{O}_{\text {all }}$ as

$$
R_{\mathbb{O} ; \tilde{\mathbb{O}}}(\mathcal{E}):=\inf \left\{1+r \mid \frac{\mathcal{E}+r \mathcal{M}}{1+r} \in \mathbb{O}, \mathcal{M} \in \tilde{\mathbb{O}}\right\},
$$

where $\tilde{\mathbb{O}} \subseteq \mathbb{O}_{\text {all }}$ is some set of channels containing $\mathbb{O}$. The extreme case $\tilde{\mathbb{O}}=\mathbb{O}_{\text {all }}$ is known as generalized robustness $[14,72,73]$ and corresponds to the max-relative entropy [74], hence we denote it by $R_{\max , \mathbb{Q}}:=R_{\mathbb{O} ; \mathbb{Q}_{\text {all }}}$. The other case of interest is the standard robustness $R_{s, \mathbb{Q}}:=$ $R_{\mathbb{O} ; \mathbb{O}}[30,41]$. We also define the smooth robustness $R_{\mathbb{O} ; \tilde{\mathbb{O}}}^{\epsilon}(\mathcal{E}):=\min \left\{R_{\mathbb{O} ; \tilde{\mathbb{O}}}\left(\mathcal{E}^{\prime}\right) \mid F\left(\mathcal{E}^{\prime}, \mathcal{E}\right) \geq 1-\epsilon, \mathcal{E}^{\prime} \in \mathbb{O}_{\text {all }}\right\}$ for $0 \leq \epsilon \leq 1$. The other type of measure, based on the hypothesis testing relative entropy $[69,70,75,76]$, is defined for $\mathcal{E} \in \mathbb{O}_{\text {all }}$ as

$R_{H, \mathbb{O}}^{\epsilon}(\mathcal{E}):=\min _{\mathcal{M} \in \mathbb{O}} \max _{\psi} R_{H}^{\epsilon}(\mathrm{id} \otimes \mathcal{E}(\psi) \| \mathrm{id} \otimes \mathcal{M}(\psi))$,

where $R_{H}^{\epsilon}(\rho \| \sigma):=\max _{\operatorname{Tr}(P \rho) \geq 1-\varepsilon}^{0 \leq P \leq 1} \operatorname{Tr}(P \sigma)^{-1}$ and the optimization is restricted to pure input states $\psi$ without loss of generality. This entropic quantity characterizes the distinguishability between $\mathcal{E}$ and the channels in the set $\mathbb{O}$. The case of $\epsilon=0$ is known as the min-relative entropy [74], denoted as $R_{\min , \mathbb{O}}(\mathcal{E})$.

It is also useful to introduce two classifications for the given theory depending on the properties of $\mathbb{O}$. We say that $\mathbb{O}$ is full dimensional if $\operatorname{span}(\mathbb{O})$ contains all channels in $\mathbb{O}_{\text {all }}$, and reduced dimensional otherwise [18]. Intuitively, in full-dimensional theories, the set $\mathbb{O}$ is of full measure, meaning that $R_{s, \mathbb{O}}(\mathcal{E})<\infty$ for all $\mathcal{E}$; examples include the theory of entanglement or local operations and shared randomness. On the other hand, reduced-dimensional theories are equipped with a set of free channels $\mathbb{O}$ of zero measure, and the standard robustness $R_{s, \mathbb{Q}}$ can diverge, examples of which include the theory of coherence, 
asymmetry, and quantum thermodynamics. In order to characterize such resources, we will often need to consider an optimization with respect to aff $(\mathbb{O})$, the affine hull of $\mathbb{O}$ $[18,77,78]$, and we define, in particular, $R_{H, \text { aff(}(\mathbb{O})}$ (respectively, $\left.R_{\min , \text { aff }(\mathbb{O})}\right)$ as the hypothesis testing entropy (minrelative entropy) minimized over $\operatorname{aff}(\mathbb{O})$ instead of $\mathbb{O}$.

We can now state our main results that connect the resource monotones with general one-shot resource transformations.

Theorem 1.-Let $\mathcal{E} \in \mathbb{O}_{\text {all }}$ and $\mathcal{N} \in \mathbb{O}_{\text {all }}^{\prime}$. If there exists a free superchannel $\Theta \in \mathbb{S}$ such that $F(\Theta(\mathcal{E}), \mathcal{N}) \geq 1-\epsilon$, then for any monotone $\mathfrak{R}_{\mathbb{O}}$ it holds that $\mathfrak{R}_{\mathbb{O}}(\mathcal{E}) \geq \mathfrak{R}_{\mathbb{O}^{\prime}}^{\epsilon}(\mathcal{N})$, as well as that $\mathfrak{R}_{\mathbb{O}}^{\delta}(\mathcal{E}) \geq \mathfrak{R}_{\mathbb{O}^{\prime}}^{2(\sqrt{\delta}+\sqrt{\epsilon})}(\mathcal{N})$ for any $0 \leq \delta \leq 1$ where $\mathfrak{R}_{\mathbb{O}}^{\epsilon}(\mathcal{E}):=\min \left\{\Re_{\mathbb{O}}\left(\mathcal{E}^{\prime}\right) \mid F\left(\mathcal{E}^{\prime}, \mathcal{E}\right) \geq 1-\epsilon, \mathcal{E}^{\prime} \in \mathbb{O}_{\text {all }}\right\}$.

Conversely, for any choices of $\epsilon, \delta \geq 0$ such that $\epsilon+2 \delta<1$, there exists a free superchannel $\Theta \in \mathbb{S}$ such that $F(\Theta(\mathcal{E}), \mathcal{N}) \geq 1-\epsilon-2 \delta$ if $R_{H, \mathbb{O}}^{\delta}(\mathcal{E}) \geq R_{s, \mathbb{Q}^{\prime}}^{\epsilon}(\mathcal{N})$ or if $R_{H, \text { aff }(\mathbb{O})}^{\delta}(\mathcal{E}) \geq R_{\text {max, } \mathbb{O}^{\prime}}^{\epsilon}(\mathcal{N})$.

Here, the parameters $\epsilon, \delta$ can be used to study the tradeoffs between the error allowed in the transformation and the values of the smoothed monotones $\mathfrak{R}_{\mathbb{Q}}^{\epsilon}$ of both the input and the output channel.

Notice that we provided two alternative achievability conditions: one using the hypothesis testing measure $R_{H, \mathbb{O}}$ and the standard robustness $R_{s, \mathbb{O}^{\prime}}$, and one using the affine hypothesis testing measure $R_{H, \text { aff(C) }}$ and the robustness $R_{\max , \mathbb{Q}^{\prime}}$. The reason for this is that the former condition will typically trivialize in reduced-dimensional theories, while the latter condition trivializes for full-dimensional theories.

Theorem 1 establishes the conditions for general resource transformation universally applicable to any resource theory, including ones with specific structures of allowed channels, reflected by choosing appropriate sets $\mathbb{O}_{\text {all }}$ and $\mathbb{O}_{\text {all }}^{\prime}$. In the special case of manipulating quantum states (channels with trivial input), we recover the results of Ref. [20] and extend them to reduced-dimensional theories. We stress that the monotones are all convex optimization problems and they reduce to computable semidefinite programs when $\mathbb{Q}$ is characterized by semidefinite constraints [58].

Distillation and dilution.-Two of the most important classes of resource transformation tasks are resource distillation, where general resources are transformed into some reference target resources, and dilution, where the reference target resources are used to synthesize a given channel through free transformations. In particular, one is often interested in two quantities: the distillable resource $d_{\mathbb{O}}^{\epsilon}(\mathcal{E})$ and the resource cost $c_{\mathbb{D}}^{\epsilon}(\mathcal{E})$; choosing a suitable class of target reference channels $\mathbb{T} \subseteq \mathbb{O}_{\text {all }}^{\prime}$, we can define

$$
\begin{aligned}
& d_{\mathbb{O}}^{\epsilon}(\mathcal{E}):=\sup \left\{\Re_{\mathbb{O}^{\prime}}(\mathcal{T}) \mid F(\Theta(\mathcal{E}), \mathcal{T}) \geq 1-\epsilon, \mathcal{T} \in \mathbb{T}, \Theta \in \mathbb{S}\right\}, \\
& c_{\mathbb{O}}^{\epsilon}(\mathcal{E}):=\inf \left\{\Re_{\mathbb{O}^{\prime}}(\mathcal{T}) \mid F(\mathcal{E}, \Theta(\mathcal{T})) \geq 1-\epsilon, \mathcal{T} \in \mathbb{T}, \Theta \in \mathbb{S}\right\},
\end{aligned}
$$

where $\mathfrak{R}_{\mathbb{O}^{\prime}}$ refers to any chosen monotone-for example, $R_{\min , \mathbb{O}^{\prime}}, R_{\max , \mathbb{O}^{\prime}}$, or $R_{s, \mathbb{O}^{\prime}}$. In the discussion below, we will fix $\Re_{\mathbb{O}^{\prime}}=R_{\text {min, } \mathbb{O}^{\prime}}$ for simplicity. The target channels $\mathbb{T}$ are often chosen as multiple copies of some fixed reference channel, but we allow for broader types of targets.

Notably, under suitable conditions on the reference channels $\mathbb{T}$, the necessary and sufficient conditions of Theorem 1 coincide, yielding a precise characterization of the resource cost.

Corollary 2.-If the chosen reference set satisfies $R_{\text {min, } \mathbb{O}^{\prime}}(\mathcal{T})=R_{s, \mathbb{O}^{\prime}}(\mathcal{T}) \forall \mathcal{T} \in \mathbb{T}$, then it holds that $c_{\mathbb{O}}^{\epsilon}(\mathcal{E})=\left\lceil R_{s, \mathbb{Q}}^{\epsilon}(\mathcal{E})\right\rceil_{\mathbb{T}}$.

Similarly, if the chosen reference set obeys $R_{\text {min,aff }\left(\mathbb{O}^{\prime}\right)}(\mathcal{T})=R_{\text {max, } \mathbb{O}^{\prime}}(\mathcal{T}) \forall \mathcal{T} \in \mathbb{T}$, then it holds that $c_{\mathbb{O}}^{\epsilon}(\mathcal{E})=\left\lceil R_{\text {max.@ }}^{\epsilon}(\mathcal{E})\right\rceil_{\mathrm{T}}$.

Here, we used the notation $\left\lceil\cdot \eta_{\pi}\right.$ to indicate the smallest value greater than or equal to the argument for which there exists a corresponding channel $\mathcal{T} \in \mathbb{T}$-this is required, for instance, when $\mathbb{T}$ forms a discrete set (see Ref. [58]).

The result establishes an operational meaning of the measures $R_{s, \mathbb{Q}}^{\epsilon}$ or $R_{\max , \mathbb{Q}}^{\epsilon}$ as long as the conditions are satisfied. This raises the question: when does a choice of reference channels $\mathbb{T}$ satisfying $R_{\min , \mathbb{Q}}(\mathcal{T})=R_{s, \mathbb{O}}(\mathcal{T})$ or $R_{\text {min,aff( }(\mathbb{O})}(\mathcal{T})=R_{\max , \mathbb{O}}(\mathcal{T})$ exist? It emerges that this is a commonly occurring feature of resource theories. For instance, it is satisfied by relevant choices of target channels in resource theories of quantum memories and communication, immediately providing a characterization of one-shot simulation cost of channels. When discussing the transformations of quantum states, it was shown that in any convex resource theory there exists maximal "golden states" $\phi_{\text {gold }}$ such that $R_{\min , \mathbb{Q}}\left(\phi_{\text {gold }}\right)=R_{\text {max, } \mathbb{Q}}\left(\phi_{\text {gold }}\right)$ [18], and indeed such states satisfy all requirements of the Corollary in theories such as quantum entanglement or quantum coherence. In the case of quantum state manipulation, our result recovers the considerations of Ref. [16], where a general framework for quantum state resources was established.

We now turn to the case of distillation. Here, we can improve the bound in Theorem 1 and obtain an alternative necessary condition. Importantly, distillation is often understood as the purification of noisy resources, in which case it is natural to consider pure reference channels $\mathbb{T}$-for instance, if the input space and output spaces coincide, unitary channels can serve as targets, while if the input space is trivial, then pure-state preparation channels can be regarded as targets. The property needed for $\mathcal{N}$ to serve as the reference resource is that the output states for pure input states remain pure. In such cases, we obtain the following general conditions.

Theorem 3.-Let $\mathcal{E} \in \mathbb{O}_{\text {all }}, \mathcal{N} \in \mathbb{O}_{\text {all }}^{\prime}$ and suppose id $\otimes \mathcal{N}(\psi)$ is pure for any pure state $\psi$. If there exists a free superchannel $\Theta \in \mathbb{S}$ such that $F(\Theta(\mathcal{E}), \mathcal{N}) \geq 1-\epsilon$, then it holds that $R_{H, \mathbb{O}}^{\epsilon}(\mathcal{E}) \geq R_{\min , \mathbb{Q}^{\prime}}(\mathcal{N})$ and $R_{H, \text { aff }(\mathbb{O})}^{\epsilon}(\mathcal{E}) \geq$ $R_{\min , \text { aff }\left(\mathbb{O}^{\prime}\right)}(\mathcal{N})$. 
Conversely, there exists a free superchannel $\Theta \in \mathbb{S}$ such that $F(\Theta(\mathcal{E}), \mathcal{N}) \geq 1-\epsilon-\delta$ if $R_{H, \mathbb{Q}}^{\epsilon}(\mathcal{E}) \geq R_{s, \mathbb{O}^{\prime}}^{\delta}(\mathcal{N})$ or if $R_{H, \text { aff }(\mathbb{O})}^{\epsilon}(\mathcal{E}) \geq R_{\max , \mathbb{O}^{\prime}}^{\delta}(\mathcal{N})$.

Theorem 3 adds a useful alternative characterization for distillation to the general condition provided by Theorem 1 . In particular, similarly to the case of dilution, we can obtain the following.

Corollary 4.-Consider any reference set $\mathbb{T}$ such that id $\otimes \mathcal{T}(\psi)$ is pure for any pure $\psi$ and any $\mathcal{T} \in \mathbb{T}$.

If $\mathbb{T}$ also satisfies $R_{\min , \mathbb{Q}}(\mathcal{T})=R_{s, \mathbb{Q}}(\mathcal{T}) \forall \mathcal{T} \in \mathbb{T}$, then it holds that $d_{\mathbb{O}}^{\epsilon}(\mathcal{E})=\left\lfloor R_{H, \mathbb{O}}^{\epsilon}(\mathcal{E})\right\rfloor_{\mathrm{T}}$.

Similarly, if the chosen reference set obeys $R_{\text {min,aff }(\mathbb{O})}(\mathcal{T})=R_{\text {max }, \mathbb{Q}}(\mathcal{T}) \forall \mathcal{T} \in \mathbb{T}$, then it holds that $d_{\mathbb{O}}^{\epsilon}(\mathcal{E})=\left\lfloor R_{H, \mathrm{aff}(\mathbb{O})}^{\epsilon}(\mathcal{E})\right\rfloor_{\mathrm{T}}$.

This establishes a precise characterization of distillable resource in any resource theory for suitable target channels $\mathbb{T}$, and furthermore gives an exact operational meaning to the resource measures $R_{H, \mathbb{O}}^{\epsilon}$ and $R_{H \text {,aff(}(\mathbb{O})}^{\epsilon}$ in the task of distillation. When the manipulated objects are quantum states, we recover the results of Refs. [16,18].

In some cases, distillation with the desired precision might not be possible. It is then of interest to instead ask how close one can approximate the chosen target channel, that is, characterize the maximal achievable fidelity of distillation. We can adapt our methods to obtain close upper and lower bounds for this quantity. Importantly, the bounds become tight for relevant reference states obeying conditions as in Corollary 4, allowing us to provide an exact expression for the fidelity of distillation. We discuss the full details in Ref. [58].

Another bound on distillation fidelity was recently presented with respect to the so-called resource weight $[22,23]$. Our approach allows one to extend the insight from these works to theories with arbitrary channel structures $\mathbb{O}_{\text {all }}$, enabling an operational application of the corresponding weight measures, some of which were previously introduced in other contexts $[49,79-83]$.

One can obtain additional results in the characterization of distillation and dilution in special cases of resource theories, for example, when the given theory is concerned with an underlying state-based resource. We discuss such cases in Ref. [58].

Quantum communication.-A central problem in quantum communication is to manipulate a given channel to enhance its communication capabilities using resources available to both parties. Quantum capacity [84-86] and simulation cost [86-88] are important figures of merit to evaluate the operational capability of quantum channels, and their one-shot characterization received considerable attention recently $[29,89-93]$. These tasks are precisely channel distillation and dilution where the reference resource is the identity channel, and the sets of free channels and free superchannels specify the accessible resources for the sender and receiver. Formally, we define the one-shot quantum capacity and simulation cost with the set of free superchannels $\mathbb{S}$ as

$$
\begin{aligned}
& Q_{\mathbb{S}}^{\epsilon}(\mathcal{E}):=\max \left\{\log d \mid \exists \Theta \in \mathbb{S}, F\left(\Theta(\mathcal{E}), \operatorname{id}_{d}\right) \geq 1-\epsilon\right\}, \\
& C_{\mathbb{S}}^{\epsilon}(\mathcal{E}):=\min \left\{\log d \mid \exists \Theta \in \mathbb{S}, F\left(\Theta\left(\operatorname{id}_{d}\right), \mathcal{E}\right) \geq 1-\epsilon\right\} .
\end{aligned}
$$

We can then use our results to immediately obtain an exact characterization of these quantities in relevant settings. For example, when $\mathbb{O}$ is the set of separable channels $\mathbb{O}_{\text {SEP }}$ whose Choi states are separable, this setting corresponds to communication with separabilitypreserving codes $\mathbb{S}=\mathbb{S}_{\mathrm{SEP}}$. This is a full-dimensional theory and it holds that $R_{\min , \mathbb{O}_{\mathrm{SEP}}}\left(\mathrm{id}_{d}\right)=R_{s, \mathbb{Q}_{\mathrm{SEP}}}\left(\mathrm{id}_{d}\right)=d$ [41]. Then, Corollaries 2 and 4 provide a complete characterization of one-shot quantum capacity and simulation cost as $Q_{S_{\mathrm{SEP}}}^{\epsilon}(\mathcal{E})=\log \left\lfloor R_{H, \mathbb{O}_{\mathrm{SEP}}}^{\epsilon}(\mathcal{E})\right\rfloor$ and $C_{\mathbb{S}_{\mathrm{SEP}}}^{\epsilon}(\mathcal{E})=$ $\log \left\lceil R_{s, \mathbb{O}_{\mathrm{SEP}}}^{\epsilon}(\mathcal{E})\right\rceil$, the latter of which recovers a result of Ref. [41]. Similar results apply to the setting of communication assisted by codes preserving the positivity of the partial transpose (PPT), where $\mathbb{O}_{\mathrm{PPT}}$ is the set of PPT channels [89,94]. We analogously obtain $Q_{\mathrm{S}_{\mathrm{PPT}}}^{\epsilon}(\mathcal{E})=\log \left\lfloor R_{H, \mathbb{O}_{\mathrm{PPT}}}^{\epsilon}(\mathcal{E})\right\rfloor$ and $C_{\mathbb{S}_{\mathrm{PPT}}}^{\epsilon}(\mathcal{E})=\log \left\lceil R_{s, \mathbb{O}_{\mathrm{PPT}}}^{\epsilon}(\mathcal{E})\right\rceil$. Interestingly, $R_{H, \mathbb{O}_{\mathrm{PPT}}}^{\epsilon}$ appeared as a bound in Ref. [90].

Another example is the case when $\mathbb{O}$ is the set of replacement channels $\mathbb{O}_{R}:=\left\{\mathcal{R}_{\sigma} \mid \mathcal{R}_{\sigma}(\cdot)=\operatorname{Tr}(\cdot) \sigma\right\}$, where $\mathbb{S}$ becomes the set of channel transformations assisted by no-signaling $(\mathrm{NS})$ correlations, $\mathbb{S}_{\mathrm{NS}}[29,95]$. Since $\mathbb{O}_{R}$ is closed under linear combinations, it is reduced dimensional. We also have $R_{\min , \operatorname{aff}\left(\mathbb{O}_{R}\right)}\left(\mathrm{id}_{d}\right)=R_{\max , \mathbb{O}_{R}}\left(\operatorname{id}_{d}\right)=$ $d^{2}$, and our results immediately give $Q_{\mathbb{S}_{\mathrm{NS}}}^{\epsilon}(\mathcal{E})=$ $\frac{1}{2} \log \left\lfloor R_{H, \text { aff }\left(\mathbb{O}_{R}\right)}^{\epsilon}(\mathcal{E})\right\rfloor$ and $C_{\mathbb{S}_{\mathrm{NS}}}^{\epsilon}(\mathcal{E})=\frac{1}{2} \log \left\lceil R_{\max , \mathbb{O}_{R}}^{\epsilon}(\mathcal{E})\right\rceil$. The one-shot NS-assisted quantum capacity was obtained in Ref. [91] in the form of a semidefinite program; our result identifies it with the affine hypothesis testing relative entropy, providing an operational meaning to this resource measure. The one-shot NS simulation cost was obtained in Refs. [29,92], which is recovered by our general approach as a special case. Furthermore, we can quantify exactly the fidelity of NS-assisted coding [58], recovering a result of Ref. [89].

Our methods apply also to the study of the entanglement of bipartite channels [34,96], where the target resources are maximally entangled states in the underlying statebased resource theory. We then establish exact one-shot rates of channel manipulation under PPT- and separabilitypreserving superchannels (see Ref. [58]).

Nonlocality and contextuality.-Quantum nonlocality has been a major subject of study not only as a key feature of quantum theory, but also as a useful resource in a number of operational tasks [97-102]. The latter view motivates a precise understanding of the manipulation of nonlocal resources [49,55,103,104], but the characterization of such one-shot transformations has 
remained elusive. Our framework encompasses this scenario by choosing $\mathbb{O}_{\text {all }}$ to be the set of no-signaling channels, where classical input or output systems are represented through dephasing in a given basis, and taking $\mathbb{O}$ to be the channels that can be constructed by local operations and shared randomness. This includes not only the standard setting of Bell nonlocality (where such channels are the classical "boxes"), but also more general resources such as steering (where channels represent "assemblages") [53,54]. Our results then provide an operational application of nonlocality measures individually introduced in different settings of nonlocality [49,105] and unified in Refs. [53,54], which we relate to one-shot resource transformations (see also Ref. [50]). We also introduce new monotones to this setting, which can add further insights into feasible manipulation of nonlocal resources. For instance, since a noisy box of the form $\mathcal{B}=(1-\epsilon) \mathcal{B}_{\mathrm{PR}}+\epsilon \mathcal{B}_{\text {free }}$, where $\mathcal{B}_{\mathrm{PR}}$ is the PopescuRohrlich (PR) box [106] and $\mathcal{B}_{\text {free }} \in \mathbb{O}$ is some local box, has $R_{\text {min, } \mathbb{O}}\left(\mathcal{B}^{\otimes n}\right)=1 \forall n$, we recover the fact that it cannot be distilled to other fundamental resources such as the PR box even when multiple copies of the box $\mathcal{B}$ are available $[103,107]$. In Ref. [58], we numerically evaluate the resource measures for a special class known as isotropic boxes [108] where we explicitly observe this property.

Notably, our framework can also be applied to another related phenomenon known as quantum contextuality, which also serves as an operational resource [109-115]. Namely, we consider the set of all classical-classical channels for consistent boxes [81] as $\mathbb{O}_{\text {all }}$ and the set of channels corresponding to noncontextual boxes as $\mathbb{O}$. Our results characterize the exact and approximate box transformations with operations that do not create contextuality, offering a new perspective to the recent resource-theoretic framework [51,52,116], as well as providing operational application of the robustness of contextuality [117-119], contextual fraction $[81,82,120]$, and hypothesis testing measures introduced in this work.

Measurement incompatibility.-Measurement incompatibility refers to the impossibility of simultaneous measurement and is closely related to the aforementioned phenomena such as Bell nonlocality and steering $[83,121,122]$. The set of POVMs $\left\{M_{a \mid x}\right\}$, where $M_{a \mid x}$ is the POVM element with outcome $a$ for the measurement labeled by setting $x$, is called compatible (or jointly measurable) if there exists a parent measurement $\left\{P_{i}\right\}$ and a conditional probability distribution $\{q(a \mid x, i)\}$ such that $M_{a \mid x}=\sum_{i} q(a \mid x, i) P_{i}$.

Our formalism can handle scenarios where resources take the form of ensembles by incorporating the classical labels of the ensembles into the description of $\mathbb{O}_{\text {all }}$. In the case of measurement incompatibility, $\mathbb{O}_{\text {all }}$ represents the set of channels corresponding to POVMs, while $\mathbb{O} \subseteq \mathbb{O}_{\text {all }}$ represents the set of compatible POVMs (see Ref. [58]). This form allows one to apply our results to this setting and characterize the approximate one-shot transformation of incompatible sets of measurements, complementing the previous works which focused on exact transformation with smaller sets of free operations in different approaches $[123,124]$ and providing operational applications of the related measures [83,125-127]. Although the robustness and weight measures are usually defined at the level of POVMs, we show in Ref. [58] that they coincide with the channel-based measures defined in our framework, allowing one to carry over the previous analyses to characterize resource transformations. We also note that the discussion here can be straightforwardly extended to channel incompatibility $[57,128]$, which includes measurement incompatibility as a special case.

Conclusions.-We established fundamental bounds on the transformations between general dynamical quantum resources in the one-shot regime. We tightly characterized the ability to manipulate resources by providing conditions for convertibility in terms of the robustness and hypothesis testing measures. In particular, under suitable assumptions, we established an exact quantification of the one-shot distillable resource and one-shot resource cost of general channels, giving a precise operational interpretation to the considered monotones in these important tasks. This not only extends and unifies previous specialized results $[34,36,41,89,91,96]$, but also sheds light on the general structure of dynamical resource theories by providing a common description of their operational aspects. Besides contributing to the theory of quantum resources, our methods find direct practical use, as we exemplified with several explicit applications.

We thank David Schmid for sharing useful references with us, which helped us expand the scope of our results. We are also grateful to the authors of Refs. [129,130] for coordinating the submission of our works. B. R. was supported by the Presidential Postdoctoral Fellowship from Nanyang Technological University, Singapore. R. T. acknowledges the support of NSF, ARO, IARPA, AFOSR, the Takenaka Scholarship Foundation, the National Research Foundation (NRF) Singapore, under its NRFF Fellow programme (Grant No. NRF-NRFF201602), and the Singapore Ministry of Education Tier 1 Grant No. 2019-T1-002-015. Any opinions, findings, and conclusions or recommendations expressed in this material are those of the author(s) and do not reflect the views of the National Research Foundation, Singapore.

Note added.-During the completion of this manuscript, we became aware of two related works, Ref. [129] by Kim et al. and Ref. [130] by Yuan et al., where the authors independently obtained results overlapping with some of our findings. The former work considers one-shot distillation and dilution of quantum channel entanglement, which coincides with our characterization of these tasks in Corollaries 2 and 4 (see Ref. [58]), while the latter work introduces a general framework for one-shot channel distillation and dilution which again corresponds to our Corollaries 2 and 4 for the cases of quantum channel resources. 
*Both authors contributed equally to this work.

†bartosz.regula@gmail.com

*ryuji.takagi@ntu.edu.sg

[1] M. Horodecki and J. Oppenheim, (Quantumness in the context of) Resource theories, Int. J. Mod. Phys. B 27, 1345019 (2013).

[2] B. Coecke, T. Fritz, and R. W. Spekkens, A mathematical theory of resources, Inf. Comput. 250, 59 (2016).

[3] E. Chitambar and G. Gour, Quantum resource theories, Rev. Mod. Phys. 91, 025001 (2019).

[4] R. Horodecki, P. Horodecki, M. Horodecki, and K. Horodecki, Quantum entanglement, Rev. Mod. Phys. 81, 865 (2009).

[5] A. Streltsov, G. Adesso, and M. B. Plenio, Quantum coherence as a resource, Rev. Mod. Phys. 89, 041003 (2017).

[6] G. Gour and R. W. Spekkens, The resource theory of quantum reference frames: Manipulations and monotones, New J. Phys. 10, 033023 (2008).

[7] F. G. S. L. Brandão and G. Gour, Reversible Framework for Quantum Resource Theories, Phys. Rev. Lett. 115, 070503 (2015).

[8] Z.-W. Liu, X. Hu, and S. Lloyd, Resource Destroying Maps, Phys. Rev. Lett. 118, 060502 (2017).

[9] G. Gour, Quantum resource theories in the single-shot regime, Phys. Rev. A 95, 062314 (2017).

[10] A. Anshu, M.-H. Hsieh, and R. Jain, Quantifying Resources in General Resource Theory with Catalysts, Phys. Rev. Lett. 121, 190504 (2018).

[11] B. Regula, Convex geometry of quantum resource quantification, J. Phys. A 51, 045303 (2018).

[12] L. Lami, B. Regula, X. Wang, R. Nichols, A. Winter, and G. Adesso, Gaussian quantum resource theories, Phys. Rev. A 98, 022335 (2018).

[13] R. Takagi, B. Regula, K. Bu, Z.-W. Liu, and G. Adesso, Operational Advantage of Quantum Resources in Subchannel Discrimination, Phys. Rev. Lett. 122, 140402 (2019).

[14] R. Takagi and B. Regula, General Resource Theories in Quantum Mechanics and Beyond: Operational Characterization via Discrimination Tasks, Phys. Rev. X 9, 031053 (2019).

[15] R. Uola, T. Kraft, J. Shang, X.-D. Yu, and O. Gühne, Quantifying Quantum Resources with Conic Programming, Phys. Rev. Lett. 122, 130404 (2019).

[16] Z.-W. Liu, K. Bu, and R. Takagi, One-Shot Operational Quantum Resource Theory, Phys. Rev. Lett. 123, 020401 (2019).

[17] R. Takagi and H. Tajima, Universal limitations on implementing resourceful unitary evolutions, Phys. Rev. A 101, 022315 (2020).

[18] B. Regula, K. Bu, R. Takagi, and Z.-W. Liu, Benchmarking one-shot distillation in general quantum resource theories, Phys. Rev. A 101, 062315 (2020).

[19] K. Fang and Z.-W. Liu, No-Go Theorems for Quantum Resource Purification, Phys. Rev. Lett. 125, 060405 (2020).

[20] W. Zhou and F. Buscemi, General state transitions with exact resource morphisms: A unified resource-theoretic approach, J. Phys. A 53, 445303 (2020).
[21] M. K. Vijayan, E. Chitambar, and M.-H. Hsieh, Simple bounds for one-shot pure-state distillation in general resource theories, Phys. Rev. A 102, 052403 (2020).

[22] B. Regula and R. Takagi, Fundamental limitations on distillation of quantum channel resources, Nat. Commun. 12, 4411 (2021).

[23] K. Fang and Z.-W. Liu, No-go theorems for quantum resource purification: New approach and channel theory, arXiv:2010.11822.

[24] K. Kuroiwa and H. Yamasaki, General quantum resource theories: Distillation, formation and consistent resource measures, Quantum 4, 355 (2020).

[25] B. Regula, L. Lami, G. Ferrari, and R. Takagi, Operational Quantification of Continuous-Variable Quantum Resources, Phys. Rev. Lett. 126, 110403 (2021).

[26] Y. Liu and X. Yuan, Operational resource theory of quantum channels, Phys. Rev. Research 2, 012035 (2020).

[27] Z.-W. Liu and A. Winter, Resource theories of quantum channels and the universal role of resource erasure, arXiv:1904.04201.

[28] G. Gour and A. Winter, How to Quantify a Dynamical Quantum Resource, Phys. Rev. Lett. 123, 150401 (2019).

[29] R. Takagi, K. Wang, and M. Hayashi, Application of the Resource Theory of Channels to Communication Scenarios, Phys. Rev. Lett. 124, 120502 (2020).

[30] R. Takagi, Optimal resource cost for error mitigation, arXiv:2006.12509.

[31] K. B. Dana, M. G. Díaz, M. Mejatty, and A. Winter, Resource theory of coherence: Beyond states, Phys. Rev. A 95, 062327 (2017).

[32] M. G. Díaz, K. Fang, X. Wang, M. Rosati, M. Skotiniotis, J. Calsamiglia, and A. Winter, Using and reusing coherence to realize quantum processes, Quantum 2, 100 (2018).

[33] D. Rosset, F. Buscemi, and Y.-C. Liang, Resource Theory of Quantum Memories and Their Faithful Verification with Minimal Assumptions, Phys. Rev. X 8, 021033 (2018).

[34] S. Bäuml, S. Das, X. Wang, and M. M. Wilde, Resource theory of entanglement for bipartite quantum channels, arXiv:1907.04181.

[35] G. Gour and C. M. Scandolo, Dynamical Entanglement, Phys. Rev. Lett. 125, 180505 (2020).

[36] G. Saxena, E. Chitambar, and G. Gour, Dynamical resource theory of quantum coherence, Phys. Rev. Research 2, 023298 (2020).

[37] S. Pirandola, R. Laurenza, C. Ottaviani, and L. Banchi, Fundamental limits of repeaterless quantum communications, Nat. Commun. 8, 15043 (2017).

[38] P. Faist and R. Renner, Fundamental Work Cost of Quantum Processes, Phys. Rev. X 8, 021011 (2018).

[39] T. Theurer, D. Egloff, L. Zhang, and M. B. Plenio, Quantifying Operations with an Application to Coherence, Phys. Rev. Lett. 122, 190405 (2019).

[40] T. Theurer, S. Satyajit, and M. B. Plenio, Quantifying Dynamical Coherence with Dynamical Entanglement, Phys. Rev. Lett. 125, 130401 (2020).

[41] X. Yuan, Y. Liu, Q. Zhao, B. Regula, J. Thompson, and M. $\mathrm{Gu}$, Universal and operational benchmarking of quantum memories, npj Quantum Inf. 7, 108 (2021). 
[42] X. Wang, M. M. Wilde, and Y. Su, Quantifying the magic of quantum channels, New J. Phys. 21, 103002 (2019).

[43] X. Wang and M. M. Wilde, Resource theory of asymmetric distinguishability for quantum channels, Phys. Rev. Research 1, 033169 (2019).

[44] J.S. Bell, On the Einstein Podolsky Rosen paradox, Physics 1, 195 (1964).

[45] N. Brunner, D. Cavalcanti, S. Pironio, V. Scarani, and S. Wehner, Bell nonlocality, Rev. Mod. Phys. 86, 419 (2014).

[46] S. Kochen and E. P. Specker, The problem of hidden variables in quantum mechanics, J. Math. Mech. 17, 59 (1967), https://www.jstor.org/stable/24902153.

[47] A. Cabello, S. Severini, and A. Winter, Graph-Theoretic Approach to Quantum Correlations, Phys. Rev. Lett. 112, 040401 (2014).

[48] J. Geller and M. Piani, Quantifying non-classical and beyond-quantum correlations in the unified operator formalism, J. Phys. A 47, 424030 (2014).

[49] J. I. de Vicente, On nonlocality as a resource theory and nonlocality measures, J. Phys. A 47, 424017 (2014).

[50] E. Wolfe, D. Schmid, A. B. Sainz, R. Kunjwal, and R. W. Spekkens, Quantifying bell: The resource theory of nonclassicality of common-cause boxes, Quantum 4, 280 (2020).

[51] K. Horodecki, A. Grudka, P. Joshi, W. Kłobus, and J. Lodyga, Axiomatic approach to contextuality and nonlocality, Phys. Rev. A 92, 032104 (2015).

[52] C. Duarte and B. Amaral, Resource theory of contextuality for arbitrary prepare-and-measure experiments, J. Math. Phys. 59, 062202 (2018).

[53] D. Schmid, D. Rosset, and F. Buscemi, The typeindependent resource theory of local operations and shared randomness, Quantum 4, 262 (2020).

[54] D. Rosset, D. Schmid, and F. Buscemi, Type-Independent Characterization of Spacelike Separated Resources, Phys. Rev. Lett. 125, 210402 (2020).

[55] R. Gallego and L. Aolita, Resource Theory of Steering, Phys. Rev. X 5, 041008 (2015).

[56] R. Uola, A. C. S. Costa, H. C. Nguyen, and O. Gühne, Quantum steering, Rev. Mod. Phys. 92, 015001 (2020).

[57] T. Heinosaari, T. Miyadera, and M. Ziman, An invitation to quantum incompatibility, J. Phys. A 49, 123001 (2016).

[58] See Supplemental Material at http://link.aps.org/ supplemental/10.1103/PhysRevLett.127.060402 for detailed proofs of our main results, which includes Refs. [59-64].

[59] M. Sion, On general minimax theorems, Pac. J. Math. 8, 171 (1958).

[60] C. Fuchs and J. van de Graaf, Cryptographic distinguishability measures for quantum-mechanical states, IEEE Trans. Inf. Theory 45, 1216 (1999).

[61] D. Kretschmann and R. F. Werner, Tema con variazioni: Quantum channel capacity, New J. Phys. 6, 26 (2004).

[62] N. Johnston, C.-K. Li, S. Plosker, Y.-T. Poon, and B. Regula, Evaluating the robustness of $k$-coherence and $k$ entanglement, Phys. Rev. A 98, 022328 (2018).

[63] F. G. S. L. Brandão and M. B. Plenio, A reversible theory of entanglement and its relation to the second law, Commun. Math. Phys. 295, 829 (2010).
[64] F. G. S. L. Brandão and N. Datta, One-shot rates for entanglement manipulation under non-entangling maps, IEEE Trans. Inf. Theory 57, 1754 (2011).

[65] G. Chiribella, G. M. D'Ariano, and P. Perinotti, Transforming quantum operations: Quantum supermaps, Europhys. Lett. 83, 30004 (2008).

[66] V. P. Belavkin, G. M. D’Ariano, and M. Raginsky, Operational distance and fidelity for quantum channels, J. Math. Phys. 46, 062106 (2005).

[67] A. Gilchrist, N. K. Langford, and M. A. Nielsen, Distance measures to compare real and ideal quantum processes, Phys. Rev. A 71, 062310 (2005).

[68] R. Renner, Security of quantum key distribution, arXiv: quant-ph/0512258.

[69] X. Yuan, Hypothesis testing and entropies of quantum channels, Phys. Rev. A 99, 032317 (2019).

[70] G. Gour and M. M. Wilde, Entropy of a quantum channel, Phys. Rev. Research 3, 023096 (2021).

[71] G. Vidal and R. Tarrach, Robustness of entanglement, Phys. Rev. A 59, 141 (1999).

[72] M. Steiner, Generalized robustness of entanglement, Phys. Rev. A 67, 054305 (2003).

[73] A. W. Harrow and M. A. Nielsen, Robustness of quantum gates in the presence of noise, Phys. Rev. A 68, 012308 (2003).

[74] N. Datta, Min- and max-relative entropies and a new entanglement monotone, IEEE Trans. Inf. Theory 55, 2816 (2009).

[75] F. Buscemi and N. Datta, Distilling entanglement from arbitrary resources, J. Math. Phys. 51, 102201 (2010).

[76] L. Wang and R. Renner, One-Shot Classical-Quantum Capacity and Hypothesis Testing, Phys. Rev. Lett. 108, 200501 (2012).

[77] B. Regula, K. Fang, X. Wang, and G. Adesso, One-Shot Coherence Distillation, Phys. Rev. Lett. 121, 010401 (2018).

[78] The affine hull aff(OD) is the smallest affine subspace which contains $\mathbb{O}$.

[79] M. Lewenstein and A. Sanpera, Separability and Entanglement of Composite Quantum Systems, Phys. Rev. Lett. 80, 2261 (1998).

[80] P. Skrzypczyk, M. Navascués, and D. Cavalcanti, Quantifying Einstein-Podolsky-Rosen Steering, Phys. Rev. Lett. 112, 180404 (2014).

[81] A. Grudka, K. Horodecki, M. Horodecki, P. Horodecki, R. Horodecki, P. Joshi, W. Kłobus, and A. Wójcik, Quantifying Contextuality, Phys. Rev. Lett. 112, 120401 (2014).

[82] S. Abramsky, R. S. Barbosa, and S. Mansfield, Contextual Fraction as a Measure of Contextuality, Phys. Rev. Lett. 119, 050504 (2017).

[83] M.F. Pusey, Verifying the quantumness of a channel with an untrusted device, J. Opt. Soc. Am. B 32, A56 (2015).

[84] C. H. Bennett, D. P. DiVincenzo, J. A. Smolin, and W. K. Wootters, Mixed-state entanglement and quantum error correction, Phys. Rev. A 54, 3824 (1996).

[85] S. Lloyd, Capacity of the noisy quantum channel, Phys. Rev. A 55, 1613 (1997).

[86] C. H. Bennett, P. W. Shor, J. A. Smolin, and A. V. Thapliyal, Entanglement-assisted capacity of a quantum 
channel and the reverse Shannon theorem, IEEE Trans. Inf. Theory 48, 2637 (2002).

[87] C. H. Bennett, I. Devetak, A. W. Harrow, P. W. Shor, and A. Winter, The quantum reverse Shannon theorem and resource tradeoffs for simulating quantum channels, IEEE Trans. Inf. Theory 60, 2926 (2014).

[88] M. Berta, M. Christandl, and R. Renner, The quantum reverse Shannon theorem based on one-shot information theory, Commun. Math. Phys. 306, 579 (2011).

[89] D. Leung and W. Matthews, On the power of PPTpreserving and non-signalling codes, IEEE Trans. Inf. Theory 61, 4486 (2015).

[90] M. Tomamichel, M. Berta, and J. M. Renes, Quantum coding with finite resources, Nat. Commun. 7, 11419 (2016).

[91] X. Wang, K. Fang, and R. Duan, Semidefinite programming converse bounds for quantum communication, IEEE Trans. Inf. Theory 65, 2583 (2019).

[92] K. Fang, X. Wang, M. Tomamichel, and M. Berta, Quantum channel simulation and the channel's smooth maxinformation, IEEE Trans. Inf. Theory 66, 2129 (2020).

[93] C.-Y. Hsieh, Communication, dynamical resource theory, and thermodynamics, PRX Quantum 2, 020318 (2021).

[94] E. M. Rains, A semidefinite program for distillable entanglement, IEEE Trans. Inf. Theory 47, 2921 (2001).

[95] R. Duan and A. Winter, No-signalling-assisted zero-error capacity of quantum channels and an information theoretic interpretation of the Lovász number, IEEE Trans. Inf. Theory 62, 891 (2016).

[96] G. Gour and C. M. Scandolo, The entanglement of a bipartite channel, Phys. Rev. A 103, 062422 (2021).

[97] H. Buhrman, R. Cleve, S. Massar, and R. de Wolf, Nonlocality and communication complexity, Rev. Mod. Phys. 82, 665 (2010).

[98] T. S. Cubitt, D. Leung, W. Matthews, and A. Winter, Improving Zero-Error Classical Communication with Entanglement, Phys. Rev. Lett. 104, 230503 (2010).

[99] J. Barrett, L. Hardy, and A. Kent, No Signaling and Quantum Key Distribution, Phys. Rev. Lett. 95, 010503 (2005).

[100] A. Acín, N. Gisin, and L. Masanes, From Bell's Theorem to Secure Quantum Key Distribution, Phys. Rev. Lett. 97, 120405 (2006).

[101] S. Pironio, A. Acín, S. Massar, A. B. de la Giroday, D. N. Matsukevich, P. Maunz, S. Olmschenk, D. Hayes, L. Luo, T. A. Manning, and C. Monroe, Random numbers certified by Bell's theorem, Nature 464, 1021 (2010).

[102] U. Vazirani and T. Vidick, Fully Device-Independent Quantum Key Distribution, Phys. Rev. Lett. 113, 140501 (2014).

[103] M. Forster, S. Winkler, and S. Wolf, Distilling Nonlocality, Phys. Rev. Lett. 102, 120401 (2009).

[104] R. V. Nery, M. M. Taddei, P. Sahium, S. P. Walborn, L. Aolita, and G. H. Aguilar, Distillation of Quantum Steering, Phys. Rev. Lett. 124, 120402 (2020).

[105] M. Piani and J. Watrous, Necessary and Sufficient Quantum Information Characterization of Einstein-PodolskyRosen Steering, Phys. Rev. Lett. 114, 060404 (2015).

[106] S. Popescu and D. Rohrlich, Quantum nonlocality as an axiom, Found. Phys. 24, 379 (1994).
[107] D. D. Dukaric and S. Wolf, A limit on non-locality distillation, arXiv:0808.3317.

[108] L. Masanes, A. Acin, and N. Gisin, General properties of nonsignaling theories, Phys. Rev. A 73, 012112 (2006).

[109] A. Hameedi, A. Tavakoli, B. Marques, and M. Bourennane, Communication Games Reveal Preparation Contextuality, Phys. Rev. Lett. 119, 220402 (2017).

[110] R. Raussendorf, Contextuality in measurement-based quantum computation, Phys. Rev. A 88, 022322 (2013).

[111] M. Howard, J. Wallman, V. Veitch, and J. Emerson, Contextuality supplies the 'magic' for quantum computation, Nature 510, 351 (2014).

[112] J. Bermejo-Vega, N. Delfosse, D. E. Browne, C. Okay, and R. Raussendorf, Contextuality as a Resource for Models of quantum computation with qubits, Phys. Rev. Lett. 119, 120505 (2017).

[113] M. Kleinmann, O. Gühne, J. R. Portillo, J.-Å. Larsson, and A. Cabello, Memory cost of quantum contextuality, New J. Phys. 13, 113011 (2011).

[114] A. Cabello, M. Gu, O. Gühne, and Z.-P. Xu, Optimal Classical Simulation of State-Independent Quantum Contextuality, Phys. Rev. Lett. 120, 130401 (2018).

[115] D. Schmid and R. W. Spekkens, Contextual Advantage for State Discrimination, Phys. Rev. X 8, 011015 (2018).

[116] B. Amaral, A. Cabello, M. T. Cunha, and L. Aolita, Noncontextual Wirings, Phys. Rev. Lett. 120, 130403 (2018).

[117] H. Meng, H. Cao, and W. Wang, The robustness of contextuality and the contextuality cost of empirical models, Sci. China Phys. Mech. 59, 640303 (2016).

[118] H. Meng, H. Cao, W. Wang, Y. Fan, and L. Chen, Generalized robustness of contextuality, Entropy 18, 297 (2016).

[119] L. Li, K. Bu, and J. Wu, Contextual robustness: An operational measure of contextuality, Phys. Rev. A 101, 012120 (2020).

[120] S. Abramsky and A. Brandenburger, The sheaf-theoretic structure of non-locality and contextuality, New J. Phys. 13, 113036 (2011).

[121] M. T. Quintino, T. Vértesi, and N. Brunner, Joint Measurability, Einstein-Podolsky-Rosen Steering, and Bell Nonlocality, Phys. Rev. Lett. 113, 160402 (2014).

[122] R. Uola, T. Moroder, and O. Gühne, Joint Measurability of Generalized Measurements Implies Classicality, Phys. Rev. Lett. 113, 160403 (2014).

[123] P. Skrzypczyk, I. Šupić, and D. Cavalcanti, All Sets of Incompatible Measurements Give an Advantage in Quantum State Discrimination, Phys. Rev. Lett. 122, 130403 (2019).

[124] F. Buscemi, E. Chitambar, and W. Zhou, Complete Resource Theory of Quantum Incompatibility as Quantum Programmability, Phys. Rev. Lett. 124, 120401 (2020).

[125] E. Haapasalo, Robustness of incompatibility for quantum devices, J. Phys. A 48, 255303 (2015).

[126] R. Uola, C. Budroni, O. Gühne, and J.-P. Pellonpää, One-to-One Mapping between Steering and Joint Measurability Problems, Phys. Rev. Lett. 115, 230402 (2015). 
[127] S. Designolle, M. Farkas, and J. Kaniewski, Incompatibility robustness of quantum measurements: A unified framework, New J. Phys. 21, 113053 (2019).

[128] T. Heinosaari and T. Miyadera, Incompatibility of quantum channels, J. Phys. A 50, 135302 (2017).
[129] H.-J. Kim, S. Lee, L. Lami, and M. B. Plenio, One-shot manipulation of entanglement for quantum channels, IEEE Trans. Inf. Theory 67, 5339 (2021).

[130] X. Yuan, P. Zeng, M. Gao, and Q. Zhao, One-shot dynamical resource theory, arXiv:2012.02781. 